\title{
A propos de la reproduction comparée en France et en Suède d'une Phéophycée-Phéosporée: le Striaria attenuata
}

\author{
B. Caram ${ }^{1}$ et S. Nygren ${ }^{2}$ \\ ${ }^{1}$ Laboratoire de Biologie végétale marine; Paris, France, \\ et \\ 2 Marinbotaniska Institutionen; Göteborg, Suède
}

KURZFASSUNG: Vergleichende Untersuchungen über die Fortpflanzung von Striaria attenuata (Phaeophyceae-Phaeosporeae) aus Frankreich und Schweden. Striaria attenuata (AG.) Grev. kommt in Frankreich regelmäßig und reichlich, in Schweden dagegen nicht regelmäßig vor. Sie trägt nur unilokuläre Sporangien. Wie sich aus vergleichenden Kulturversuchen ergab, ist der Lebenszyklus dieser Alge in den beiden Arealen wesentlich verschieden. Caram (1965) wies für die Pflanzen aus Frankreich einen digenetischen, diplo-haplophasischen Generationswechsel nach $(2 \mathrm{n} \approx 20, \mathrm{n} \approx 10)$. Es können aber auch gleichartige diploide Generationen nach Verschmelzung der Schwärmer aus unilokulären Sporangien aufeinanderfolgen; der Zyklus ist dann monogenetisch und diplophasisch. Striaria attenuata kann also sowohl Sporophyt als auch Gametophyt sein, im allgemeinen ist ersteres der Fall. In Schweden scheint die Entwicklung direkt zu erfolgen. KyurN (1934) erhielt aus den Schwärmern Striaria-Pflanzen, die aber nicht fertil wurden. Die neuen Untersuchungen ergänzen Kylıns Ergebnisse. Die Schwärmer entwickelten sich direkt zu fertilen jungen Striaria-Pflanzen oder zu Mikrothalli mit unilokulären und plurilokulären Sporangien. Wenn auch der zytologische Nachweis noch aussteht, so lassen die bisher vorliegenden Beobachtungen darauf schließen, daß bei Striaria attenuata in Schweden wahrscheinlich diploide Generationen aus apomeiotischen Schwärmern aufeinanderfolgen.

\section{INTRODUCTION}

Le Striaria attenuata (AG.) Grev. est une algue brune filamenteuse de la famille des Striariacées et l'ordre des Dictyosiphonales. Sa distribution est la suivante: côtes françaises de l'Atlantique (nord) et de la Manche; côtes anglaises; côtes danoises (Kattegat nord); côtes occidentales suédoise et norvégienne. Il a également été signalé en quelques points des côtes méditerranéennes française et italienne. La plante adulte ne porte que des zoïdocystes uniloculaires.

En France, où on peut la récolter régulièrement et abondamment dans toutes ses stations, l'espèce a toujours été considérée comme printanière. En fait, elle a été draguée à plusieurs reprises dans la rade de Brest, à $20-25 \mathrm{~m}$ de profondeur, durant 
les mois de juillet et août, alors qu'elle a entièrement disparu des hautes cuvettes où elle vit au printemps. Il est vraisemblable qu'on la trouverait dans les mêmes conditions, et à la même époque, dans ses autres localités françaises. Cela signifie qu'elle existe sous sa forme délophycée pendant au moins six mois de l'année.

En Suède, par contre, l'espèce est plus rare. Elle est uniquement estivale et vit de juin à septembre à des profondeurs allant de 5 à $25 \mathrm{~m}$.

KYLIN (1934) en a fait les premières cultures, dans une eau de mer enrichie de phosphate et nitrate de sodium et de citrate de fer. Il a obtenu en un mois, par développement direct des zoïdes, de jeunes plantes de Striaria qui ne sont pas devenues fertiles. Il conclut que, par un phénomène d'apoméiose, cette algue a perdu toute sexualité.

\section{RESULTATS ET CONCLUSIONS}

Beaucoup plus tard, durant le travail de thèse de l'un de nous (Caram 1965), le Striaria attenuata a été récolté sur les côtes bretonnes et, de 1958 à 1964, il en a été fait de nombreuses cultures, toutes dans le milieu E.S.L., à une température de $10^{\circ} \mathrm{C}$ et à la lumière du jour, plus trois heures de lumière artificielle en supplément, en hiver. Les résultats ont été les suivants: (1) les thalles délophycés sont diploïdes

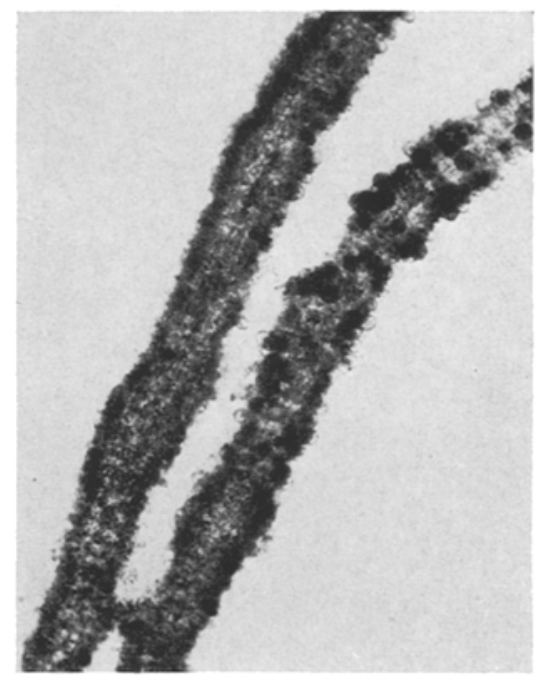

Fig. 1: Striaria attenuata (AG.) Grev. Deux thalles adultes, fertiles, obtenus en culture, en France (vue partielle). $(\times 150)$

( $2 n=20$ chromosomes environ); (2) leurs zoïdocystes sont le siège d'une méiose et libèrent des zoïdes haploïdes $(n=10$ chromosomes environ) qui sont à l'origine d'une ontogénie complexe. En effet, ces zoïdes se comportent parfois comme des spores de passage, qui engendrent des gamétophytes à zoïdocystes pluriloculaires, 
producteurs de gamètes dont la copulation donne de nouveaux sporophytes diploïdes; d'autres fois, ils se comportent comme des zoogamètes qui copulent à leur sortie du zoïdocyste, redonnant ainsi immédiatement la génération diploïde. Par conséquent, il peut y avoir un cycle long, digénétique, hétéromorphe, ou un cycle court, monogénétique, et le Striaria, en France, peut donc être à la fois un sporophyte et un gamétophyte (Fig. 1).

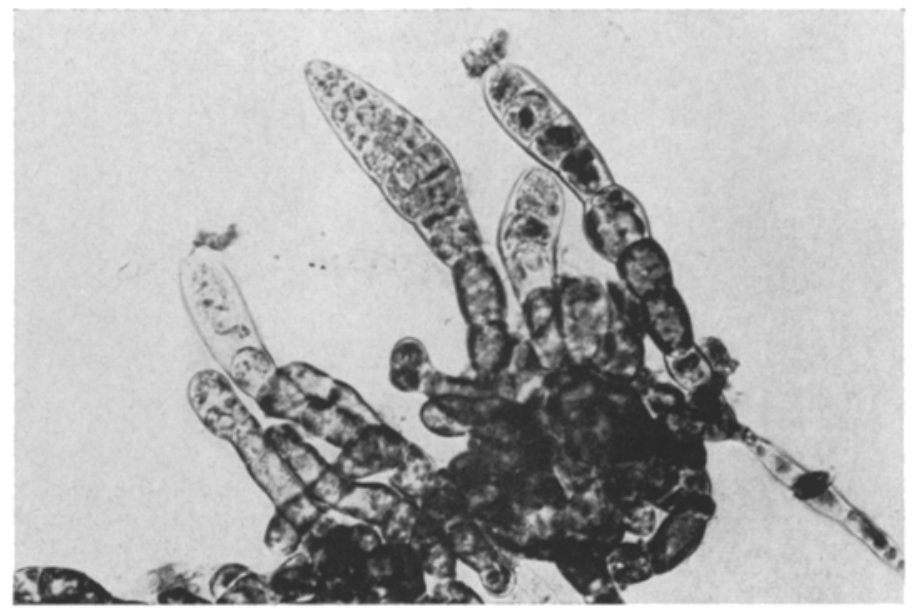

Fig. 2: Un jeune microthalle porteur de zoïdocystes pluriloculaires, des cultures du Striaria attenuata de Danemark. ( $\times$ 400)

Ces résultats, si différents de ceux de KYLIN, ont conduit à recommencer ces cultures en Suède. Pendant trois années de suite, l'espèce a été recherchée sans aucun succès, malgré dragages et plongées tout le long de la côte.

Toutefois, en 1965, un seul specimen envoyé par CHRISTEnsen de Danemark (Kattegat nord), a permis d'établir une série de cultures, toujours dans l'Erdschreiber. Aucune copulation des zoïdes n'a été observée, mais leur développement direct a donné des microthalles (Fig. 2) garnis de zoïdocystes pluriloculaires de grande taille, caractéristiques du Striaria. Ces microthalles ayant été isolés, il s'est développé dans les mêmes boîtes de Petri des sporophytes qui, dans certains cas encore très jeunes et à peine cortiqués, portaient de nombreux zoïdocystes uniloculaires (Fig. 3). On peut supposer que les zoïdes des pluriloculaires avaient copulé, mais cette copulation n'a pas été observée, pas plus que la méiose (CARAM 1966).

Enfin, l'année dernière (1968), dans le Gullmarfjord, près de la Station Zoologique de Kristineberg, le Striaria attenuata a réapparu en abondance et l'un de nous (NYGren) a pu établir, en juillet, une série de cultures, cette fois dans le milieu de von Stosch (préparé avec de l'eau de mer de salinité 30\%), à une température de $10^{\circ} \mathrm{C}$ et avec une photopériode de 14 heures en lumière artificielle (tube fluorescent blanc brillant, 1000 lux environ). Ces cultures ont donné des microthalles agglomérés en touffes denses et encore stériles après six mois. Il a fallu les dissocier avec une aiguille pour qu'ils produisent, au bount d'un mois, de jeunes Striaria qui sont devenus 
fertiles, et dont ils étaient les protonémas. Avec les zoïdes de ces jeunes Striaria il a été fait, dans les mêmes conditions, une seconde série de cultures où se sont développés d'autres microthalles, dont les uns portaient à la fois des Striaria fertiles et des zoïdocystes pluriloculaires (Fig. 4), et les autres seulement des zoïdocystes, pluriloculaires et uniloculaires (Fig. 5).

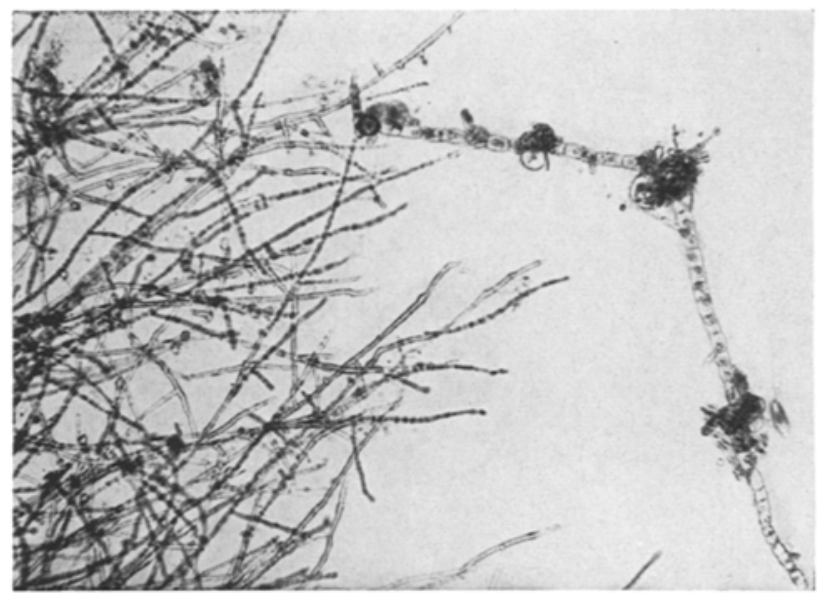

Fig. 3: Un Striaria attenuata adulte, des cultures de Danemark, très jeune, à peine cortiqué et déja fertile. Les zoïdes ont germé sur place, hérissant le thalle de filaments. $(\times 37)$

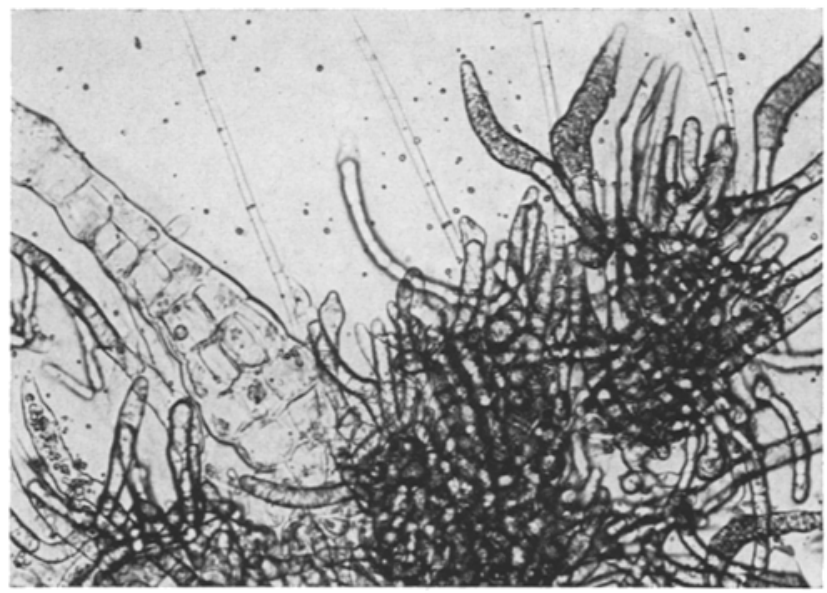

Fig. 4: Un microthalle de Striaria attenuata, des cultures de Suède, portant des zoïdocystes pluriloculaires et un thalle adulte dont on aperçoit la base. $(\times 300)$

A partir de deux specimens de microthalles stériles et de deux specimens de Striaria, provenant de la première série de cultures, de nouvelles cultures ont été menées parallèlement à Paris (CARAM). Les microthalles en touffes denses très pigmentées ont été mis: l'un dans le même milieu de von Stosch (eau de mer provenant 
de Kristineberg), à une température de $10^{\circ} \mathrm{C}$ et une photopériode de 14 heures (tube

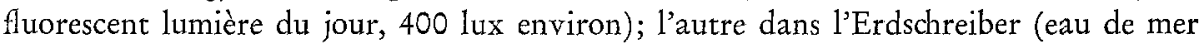
de Kristineberg également), à une température de $140 \mathrm{C}$ et exposé à la lumière du jour (environ 600 lux). Dans les deux cas, et sans dissociation, les microthalles sont devenus fertiles et ont produit, les uns des zoïdocystes pluriloculaires et des Striaria fertiles, les autres des zoïdocystes uniloculaires et pluriloculaires. Ces résultats concordent entièrement avec ceux obtenus, à peu près en même temps, en Suède.

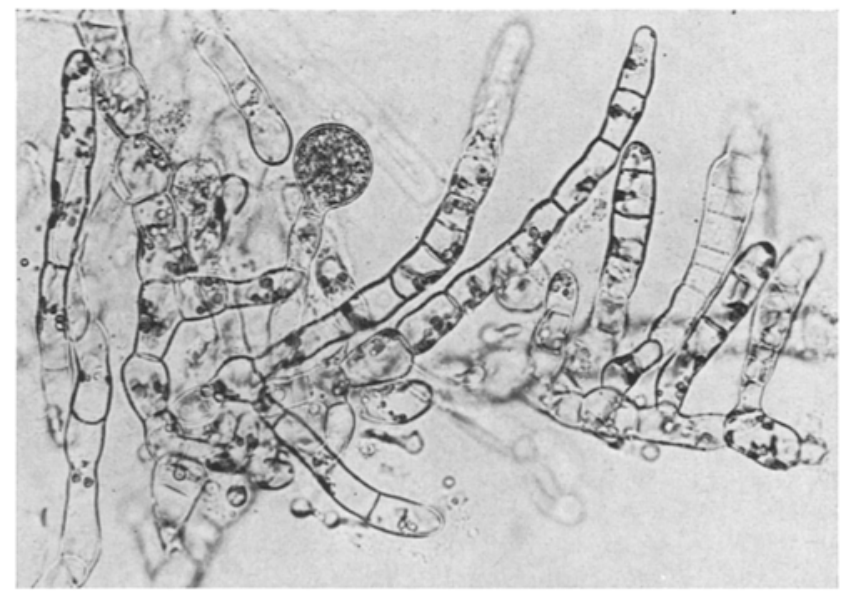

Fig. 5: Un microthalle de Striaria attenuata, des cultures de Suède, portant des zoïdocystes uni- et pluriloculaires. $(\times 600)$

Bien que plus complets que ceux de Krusn, ils démontrent que le cycle du Striaria en Suède diffère de celui qui a été observé en France.

D’après ces résultats, le développement de l'algue suédoise consiste en une succession de générations composées chacune de microthalles porteurs, soit seulement de zoïdocystes (uni-et pluriloculaires), soit de zoïdocystes (pluriloculaires) et de Striaria adultes fertiles. Plusieurs générations ont été ainsi obtenues. Les microthalles se comportent donc à la fois comme des pléthysmothalles en produisant directement des zoïdocystes, et comme des protonémas quand ils engendrent des Striaria. Malgré l'absence de données caryologiques, on peut penser avec KrLIN (1934) que tous ces thalles sont diploïdes, la méiose faisant défaut.

Peut-on expliquer ce comportement de l'algue par les conditions défavorables qui règnent sur la côte suédoise au moment où, peut-être, un déterminisme génétique déclanche la méiose? Les eaux de surface sont encore très froides au printemps et l'algue subsiste encore sous sa forme microscopique de survie dans des fonds peu éclairés. En France, on la trouve déja au début de mars dans les hautes cuvettes. L'éclairement intense qu'elle reçoit à ce moment peut-il être un élément déterminant pour provoquer les stades de méiose qui sont très nombreux alors?

Ces observations ne sont, toutefois, que préliminaires et nous nous proposons de 
faire, parallèlement toujours, de nouvelles cultures à des températures et des photopériodes plus largement variées.

\section{RESUME}

1. Le thalle adulte du Striaria attenuata ne porte que des zoïdocystes uniloculaires. Sa présence est régulière en France, mais plus irrégulière en Suède.

2. Des cultures comparées, faites dans les deux pays, ont montré des variations dans le cycle de l'algue.

3. En France, le cycle, sexué, est double: (a) digénétique, hétéromorphe, diplohaplophasique $(2 \mathrm{n}=20$ et $\mathrm{n}=10$ chromosomes environ); (b) monogénétique, diplophasique, par la copulation immédiate des zoïdes des zoïdocystes uniloculaires.

4. En Suède, le cycle, asexué, est sans doute entièrement diplophasique, la germination directe des zoïdes donnant une succession de microthalles porteurs, soit de zoïdocystes uni- et pluriloculaires seulement, soit de zoïdocystes pluriloculaires et de Striaria adultes.

\section{LITTERATURE CITEE}

CARAM, B., 1965. Recherches sur la reproduction et le cycle sexué de quelques Phéophycées. Vie Milieu (Sér. A) 16, 21-221.

- 1966. Sur la reproduction de deux Striariacées des eaux danoises. C. $r$, hebd. Séanc. Acad. Sci., Paris 262, 2333-2335.

Kylin, H., 1934. Zur Kenntnis der Entwicklungsgeschichte einiger Phaeophyceen. Lunds Univ. Arsskr. (= Acta Univ. lund. N.F.) Avd. 230 (9), 1-189.

Adresse du premier auteur: Dr. B. CARAM

Université de Paris

Faculté des Sciences

Laboratoire de Biologie végétale marine

7, Quai St. Bernard

Paris 5e, France 